This full text version, available on TeesRep, is the PDF (final version) of:

Weston, M. et. al. (2011) 'Reduction in physical match performance at the start of the second half in elite soccer', International Journal of Sports Physiology and Performance, 6 (2), pp.174-182.

For details regarding the final published version please click on the following link:

http://journals.humankinetics.com/ijspp-current-issue/ijspp-volume-6-issue-2-june/Reduction-in-PhysicalMatch-Performance-at-the-Start-of-the-Second-Half

When citing this source, please use the final published version as above.

This document was downloaded from http://tees.openrepository.com/tees/handle/10149/125997 Please do not use this version for citation purposes.

All items in TeesRep are protected by copyright, with all rights reserved, unless otherwise indicated. 


\title{
Reduction in Physical Match Performance at the Start of the Second Half in Elite Soccer
}

\author{
Matthew Weston, Alan M. Batterham, Carlo Castagna, \\ Matthew D. Portas, Christopher Barnes, \\ Jamie Harley, and Ric J. Lovell
}

\begin{abstract}
Purpose: Soccer referees' physical match performances at the start of the second half (46-60 $\mathrm{min}$ ) were evaluated in relation to both the corresponding phase of the first half (0-15 $\mathrm{min})$ and players' performances during the same match periods. Methods: Match analysis data were collected (Prozone, UK) from 12 soccer referees on 152 English Premier League matches during the 2008/09 soccer season. Physical match performance categories for referees and players were total distance, high-speed running distance (speed $>5.5 \mathrm{~m} / \mathrm{s}$ ), and sprinting distance $(>7.0 \mathrm{~m} / \mathrm{s}$ ). The referees' heart rate was recorded from the start of their warm-up to the end of the match. The referees' average distances (in meters) from the ball and fouls were also calculated. Results: No substantial differences were observed in duration (16:42 $\pm 2: 35$ vs $16: 27 \pm 1: 00 \mathrm{~min})$ or intensity ( $107 \pm 11$ vs $106 \pm 14$ beats/ $\mathrm{min}$ ) of the referees' preparation periods immediately before each half. Physical match performance was reduced during the initial phase of the second half when compared with the first half in both referees (effect sizes-standardized mean differences -0.19 to 0.73 ) and players (effect sizes 0.20 to 1.01). The degree of the decreased performance was consistent between referees and players for total distance $(4.7 \mathrm{~m})$, high-speed running $(1.5 \mathrm{~m})$, and sprinting $(1.1 \mathrm{~m})$. The referees were closer to the ball (effect size 0.52) during the opening phase the second half. Conclusion: Given the similarity in the referees' preparation periods, it may be that the reduced physical match performances observed in soccer referees during the opening stages of the second half are a consequence of a slower tempo of play.
\end{abstract}

Keywords: work rate, tempo, match play, half-time, preparation

\footnotetext{
Matthew Weston is with the Department of Sport \& Exercise Sciences, Teesside University, Middlesbrough, U.K., and the Professional Game Match Officials Limited (PGMOL), The FA Premier League, London, UK. Alan M. Batterham is with the School of Health and Social Care, Teesside University, Middlesbrough, UK. Carlo Castagna is with the School of Sport and Exercise Sciences, University of Roma, Rome, Italy. Matthew D. Portas is with the Department of Sport \& Exercise Sciences, Teesside University, Middlesbrough, UK. Christopher Barnes is with Science and Medicine Dept, West Bromwich Albion Football Club, UK. Jamie Harley is with the Department of Sport \& Exercise Sciences, Teesside University, Middlesbrough, UK. Ric J. Lovell is with the University of Hull, Kingston upon Hull, UK.
} 
The time-motion demands of elite soccer match play have been reported extensively in the literature. ${ }^{1-4}$ Segmentation of the match demands into $15 \mathrm{~min}$ periods has become commonplace, and this methodological approach has demonstrated a decrement in work performed immediately after half-time (46-60 min) in comparison with the corresponding phase of the first half $(0-15 \mathrm{~min}))^{1,2}$ The same trend has also been evident in match referees, with a significant drop in total distance covered, ${ }^{5,6}$ distance covered at high-intensity, ${ }^{5,6}$ and sidewards running. ${ }^{5,7}$

This decrease in player and referee work rate during the initial phase of the second half may be attributed to the drop in muscle temperature (approx. $1.5-2.0^{\circ} \mathrm{C}$ ) that occurs during the typically passive 15 min half-time period. ${ }^{8}$ However, referees' physiological status at the start of the second half may not be disparate to that observed before the start of the match, since prematch routines typically involve approximately $15 \mathrm{~min}$ of rest between the end of the prematch warm-up and the start of the match, as evidenced by an illustration of a typical referee's heart rate response during a Premier League match. ${ }^{9}$

It is unlikely that the reduced high-intensity performance observed during this match period is explained by dehydration ${ }^{10}$ or fatigue given that there is no betweenhalf difference for high-intensity distance covered in the subsequent period (16-30 min vs $61-75 \mathrm{~min}) .^{5,7,11}$ An alternative explanation is a comparative reduction in the tempo of the match, explained by a combination of (1) an aim to establish tactical superiority at the start of the match, (2) tactical amendments made during the half-time interval, or (3) "meso-pacing" strategies to conserve energy for later in the half. ${ }^{12}$ Although a reduced match tempo and/or implementation of a pacing strategy at the start of the second half may be difficult to discern from time-motion data, this supposition has been highlighted previously. ${ }^{7}$

One approach to determine changes in game tempo could be to examine the relationship of work-rate patterns between players and match referees. Given that previous research has shown the work rate of referees to be related in part to that of the players, ${ }^{13}$ it could be speculated that any decrement observed for referees after half-time would be of a magnitude similar to that observed for the players during the same match. With this in mind, the aim of the present study was to investigate referees' work-rate patterns immediately after half-time, in relation to both the corresponding phase of the first half and also the change in players' work rates during the same time periods.

\section{Methods}

\section{Subjects and Study Design}

Participants were 12 English Football Association (FA) Premier League referees. Data were collected for FA Premier League matches during the 2008/09 English soccer season. The mean age and body mass of the referees was $39.5 \pm 6.2$ years and $81.2 \pm 8.6 \mathrm{~kg}$, respectively. Written informed consent was received from all referees after verbal and written explanations of the observational research design. The local institutional review board approved this study design.

\section{Methodology}

Physical Match Performances. The referees' physical match performances were examined for 152 matches (mean $12.7 \pm 5.3$ matches per referee). Each match 
was examined using a computerized, semiautomatic video match-analysis image recognition system (Prozone, Leeds, UK). Recent findings have demonstrated that the Prozone match-analysis system provides valid and reliable analyses of movement patterns of footballers during match play. ${ }^{3,14}$ The objective measures of physical match performance selected for analysis were (1) total distance covered (m); (2) high-speed running distance ( $\mathrm{m}$; running speed $\left.>5.5 \mathrm{~m} \cdot \mathrm{s}^{-1}\right)$; (3) sprinting distance $\left(\mathrm{m}\right.$; running speed $\left.>7.0 \mathrm{~m} \cdot \mathrm{s}^{-1}\right)$; and for referees (4) mean distance from the ball (m); (5) mean distance from fouls (m); and (6) total number of decisions. The players' distances were calculated as the mean individual player distance (goalkeepers' data were excluded from the analysis).

To examine the relationship between the referees' and the players' match running performances, average total distance covered, high-speed running distance and sprinting distance during the initial 15-min period of first half ( $0-15 \mathrm{~min})$ and the initial period of the second half $(46-60 \mathrm{~min})$ were calculated. The referees' technical performance measures of average distance from the ball, average distance from fouls, and the total number of decisions were also calculated for these periods.

Heart Rate Analysis. The referees' warm-up before matches was standardized and consisted of low-speed running and dynamic mobility exercises followed by short high-speed and maximal running bouts of approx. $18 \mathrm{~m}$. All referees were familiarized with the protocol, as it had been part of their prematch regime for several seasons before this study. Heart rate during the warm-up and matches was recorded via short-range telemetry using Polar RS400 monitors (Polar, Kempele, Finland), with the data being recorded every second. Data recording commenced from the start of the referees' warm-up and finished upon the final whistle. The referees inserted markers into the heart rate files, using the appropriate button on the heart rate monitor, to indicate the precise moment at which (1) the warm-up started, (2) the warm-up terminated, (3) start of the first half, (4) end of the first half, (5) start of the second half, and (6) end of the second half. Following each match, the data were downloaded onto a computer for analysis using Polar Precision software (v3.0, Polar, Kempele, Finland) and the following measures were calculated:

- Prematch: the time (min:s) between the end of the referees' warm-up and the start of the first half

- Prematch heart rate: average heart rate $\left(b \cdot \mathrm{min}^{-1}\right)$ for the period between the end of the referees' warm-up and the start of the first half

- Half-time: the time (min:s) between the end of the first half and the start of the second half

- Half-time heart rate: average heart rate $\left(b \cdot \mathrm{min}^{-1}\right)$ for the period between the end of the first half and the start of the second half.

\section{Statistical Analysis}

Data are presented as the mean \pm standard deviation. Paired $t$ statistics were calculated for (1) the referees' heart rates during the prematch and half-time periods and also the duration of these periods, (2) the referees' running and technical performances during the initial $15 \mathrm{~min}$ of the first half $(0-15 \mathrm{~min})$ and the initial $15 \mathrm{~min}$ period of the second half (46-60 $\mathrm{min})$, (3) the players' running performances for 0-15 min and 46-60 min, and (4) the mean differences between the referees' and 
players' running performances for 0-15 min and 46-60 min. Effect sizes (Cohen's d) for between-group differences were determined, with values of $0.20,0.50$, and 0.80 representing a small, moderate, and large difference, respectively. ${ }^{15} \mathrm{~A}$ priori, we defined the minimal practically important difference as 0.2 between-subject standard deviations. Inference was then based on the disposition of the confidence interval for the mean difference to this smallest worthwhile effect; the probability (percent chances) that the true population difference between first and second half is substantial ( $>0.2$ SDs) or trivial was calculated as per the magnitude-based inference approach detailed by Batterham and Hopkins. ${ }^{16}$ These percent chances were qualified via probabilistic terms assigned using the following scale: ${ }^{17}<0.5 \%$, most unlikely or almost certainly not; $0.5-5 \%$, very unlikely; 5-25\%, unlikely or probably not; 25-75\%, possibly; 75-95\%, likely or probably; 95-99.5\%, very likely; $>99.5 \%$, most likely or almost certainly. Analyses were performed using the SPSS analysis software package (Version 16), with the spreadsheet of Hopkins ${ }^{18}$ being used to determine the $90 \%$ confidence intervals of the between-group differences. Analyses were conducted with the raw untransformed data, with confidence intervals for mean differences confirmed via a criterion bootstrapping technique.

\section{Results}

The referees' warm-up protocol lasted 13:40 \pm 2:40 min and elicited a mean heart rate response of $130 \pm 12 \mathrm{~b} \cdot \mathrm{min}^{-1}$. There was no substantial difference in the duration of referees' prematch period, when compared with the half-time interval (16:42 \pm $2: 35$ vs $16: 27 \pm 1: 00 \mathrm{~min}, P=.492, d=0.08)$. In addition, no substantial difference in heart rate between these two periods was observed $\left(107 \pm 11 \mathrm{vs} 106 \pm 14 \mathrm{~b} \cdot \mathrm{min}^{-1}\right.$, $P=.550, d=0.03$ ). The referees' and players' physical match performances during the initial $15 \mathrm{~min}$ of each half are summarized in Table 1 . All physical performance variables decreased substantially during the initial phase of the second half, with small effect sizes for the observed decreases in high-speed running and sprinting in both referees and players and medium and large effect sizes for the reduction in the referees' and players' total distance covered, respectively. For the referees' technical measures, a substantial difference was observed for average distance from the ball, with a moderate effect size, whereas no substantial differences were observed for the referees' average distance from fouls and the number of decisions.

The difference between referees $(-113.6 \mathrm{~m})$ and players $(-108.9 \mathrm{~m})$ in the decline in total distance covered between the first and second half periods was 4.7 $\mathrm{m}(90 \%$ confidence interval -11.0 to $21.0 \mathrm{~m})$. The percent chances that the true population difference is substantial / trivial are 1/99; very likely a trivial difference. For high-speed running distance, the difference in the decline between referees $(-11.7 \mathrm{~m})$ and players $(-10.3 \mathrm{~m})$ was $1.5 \mathrm{~m}(-4.6 \mathrm{~m}$ to $7.4 \mathrm{~m})$. The percent chances that the true population difference is substantial / trivial are 6/94; the difference is likely to be trivial. For sprinting distance, the difference in the decline between referees $(-4.4 \mathrm{~m})$ and players $(-3.4 \mathrm{~m})$ was $1.1 \mathrm{~m}(-2.7 \mathrm{~m}$ to $4.9 \mathrm{~m})$. The percent chances that the true population difference is substantial / trivial are 6/94; the difference is likely to be trivial. The difference between the referees' average distance from the ball during the first and second half periods was $-0.8 \mathrm{~m}(-0.5 \mathrm{~m}$ to -0.9 $\mathrm{m})$. The percent chances that the true difference is substantial / trivial are 100/0; the difference is almost certainly a substantial difference. 


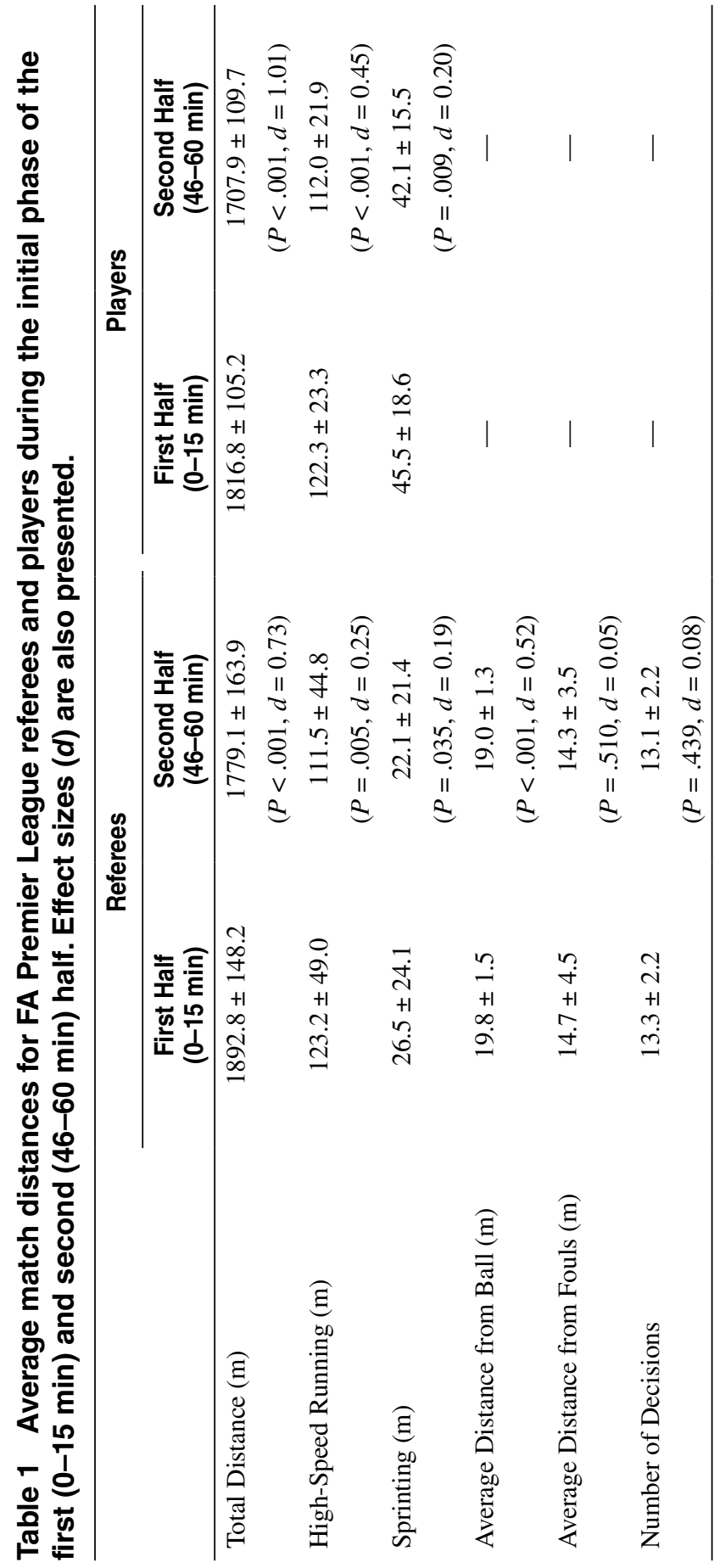




\section{Discussion}

The aim of the present study was to investigate soccer referees' work-rate patterns immediately after half-time, in relation to both the corresponding phase of the first half and also the change in players' work rates during the same time period. We found that the referees' and players' physical match performances decreased during the initial 15 min period of the second half when compared with the corresponding phase of the first half. In addition, the magnitude of the decreased physical performances was consistent between referees and players. For the referees' differences in technical performance measures were observed only for the average distance from the ball, which decreased at the start of the second half. The referees' prehalf preparation periods were consistent as no substantial differences were observed in the duration of the referees' prematch period, and also heart rates during this period, when compared with the half-time interval.

Previous research has demonstrated reduced physical match performances during the opening phase of the second half when compared with the first half in referees and players. For example, both Krustrup and Bangsbo ${ }^{5}$ and Bradley et $\mathrm{al}^{2}$ reported less high-intensity running in the first $15 \mathrm{~min}$ period of the second half when compared with the first half for referees and players, respectively. This decrease in work rate may be attributed to the drop in muscle temperature $\left(T_{m}\right)$ (approx. 1.5-2.0 $0^{\circ} \mathrm{C}$ ) that occurs during the half-time period. ${ }^{8}$ Evidence for this comes from Mohr et al, ${ }^{19}$ who, in a study examining the effect of a half-time rewarm-up on muscle temperature and sprint performance during soccer matches, reported that the decline in $\mathrm{T}_{\mathrm{m}}$ that occurs during the half-time interval is associated with a lower sprint capacity (approx. 2.4\%) at the onset of the second half. Whereas sprint performance was maintained when moderate-intensity activities performed during the half-time interval preserved $\mathrm{T}_{\mathrm{m}} \cdot{ }^{16} \mathrm{In}$ a study examining the physical demands of top-class assistant refereeing Krustrup et $\mathrm{al}^{7}$ reported that $\mathrm{T}_{\mathrm{m}}$ decreased by $1.3^{\circ} \mathrm{C}$ during the half-time interval. The authors also observed a lower amount of high-intensity running and distance covered moving sideways during the initial 5-min period of the second half, and speculated that this may have been the result of a lower tempo of match. However, the players' physical match performances were not reported and without such information it is difficult to substantiate this conclusion. The authors went on to suggest that the observed drop in $\mathrm{T}_{\mathrm{m}}$ may explain the reduced performances.

In the Krustrup et $\mathrm{al}^{7}$ study, the duration of the prematch warm-up was approximately 30 min yet no information was provided on the duration between the end of the prematch warm-up and the start of the match, whereas Mohr et a $1{ }^{19}$ reported that for players the prematch warm-up period ended $5 \mathrm{~min}$ before a noncompetitive match, a period of time consistent with that observed during competitive matches. However, due to match administration procedures before competitive matches the time interval between the end of the referees' warm-up and the start of the match may well be similar to the duration of the half-time interval. This is evidenced by the present study's findings where no substantial differences in duration and heart rate were observed for the prematch period when compared with the half-time interval. Therefore, given the similarity in preparation periods on competitive matches it may well be that the referees have similar physiological status (ie, $\mathrm{T}_{\mathrm{m}}$, $\mathrm{HR}$, oxygen consumption, metabolic acidosis) at the start of the second half as they do at the start of the first half. 
Therefore, regardless of the physiological mechanisms responsible, since referees' passive "down time" before the start of each half is similar, it is unlikely that the reduced physical performance observed in the early stages of the second half is due to the half-time interval. With this in mind, another possible explanation for the referees' reduced physical performances at the start of the second half could be a response to a slower tempo of play as opposed to one of physiological impairment.

The referees' average distance from the ball has previously been used as a measure of their ability to keep up with play. ${ }^{20}$ Weston et $\mathrm{al}^{20}$ reported an average distance from the ball of $19.3 \pm 1.1 \mathrm{~m}$ on a total of 778 English Premier League soccer matches, with a between-referee coefficient of variation of 5\% illustrating the stable nature of this variable. While there was no substantial difference in the total number of decisions or the referees' average distance from fouls between the initial $15 \mathrm{~min}$ period of the second half with the corresponding phase of the first half, the referees were closer to the ball. Therefore, these data may well support the supposition of a slower tempo of play during the initial phase of the second half in comparison with the first half. A slower tempo of play at the beginning of the second half could be explained by tactical advice from the $\operatorname{coach}^{19}$ during the half-time interval. Alternatively, given that Edwards and Noakes ${ }^{12}$ have recently proposed a model for pacing strategies to explain the self-regulation of elite soccer performance in which players behaviorally modulate their efforts according to a subconscious strategy, it may be that players employ pacing strategies to conserve energy for later in the half.

Previous research has demonstrated that referees' physical match performances are in part related to those of the players in the same match. ${ }^{13}$ Further demonstration of this relationship was observed in the present study given that the degree of the between-half change in total distance covered, high-speed running, and sprinting was consistent for both groups. Indeed, this is the first study to report on a direct relationship between referees' and players' work rates by comparing within-match work-rate periods in the same match, as previous comparisons were drawn from mean match performances. ${ }^{12}$

In summary, we found a reduction in referees' physical match performance during the initial phase of the second half when compared with the corresponding phase of the first half. The decrease was consistent with the observed reduction in player physical match performance and no differences were observed in duration and intensity of the referees' prematch and half-time intervals. This raises the possibility that the referees' reduced physical performances may have been a consequence of a slower tempo of match play during the opening phase of the second half when compared with the opening phase of the first half. This supposition is supported by the fact that the referees were closer to play at the start of the second half.

\section{Practical Applications}

Reductions in running performance, and in particular high-speed running, may not always reflect fatigue or suboptimal preparation on behalf of the match participants, but may also be a reflection of a slower tempo of match play. In addition, given the relationship between referees' and players' physical match performances it is important that referees' data are evaluated and interpreted in the context of the players' performances for the same game. Furthermore, semiautomated match analysis com- 
panies could look to develop systems whereby the tempo of the match can be evaluated in an objective manner, which would help sports scientists to interpret physical performances in the context of tactical strategies employed during competitive match play. A limitation of the current study was that even though we were comparing the referees' post half-time physical match performances to those recorded by players on the same match, the prematch "down-time" period will have been longer for the referees when compared with the players. In addition, whereas the referees did not perform any rewarm-up during the half-time interval, the activity of the players during this interval is unknown and we cannot discount that some rewarming activity may have taken place in dressing rooms and/or on the pitch immediately before the start of the second half. Nonetheless, to fully maximize the benefit of the warm-up period it may be that the time available between the end of the warm-up and the kickoff for match officials and players is addressed by soccer-governing bodies.

\section{Acknowledgments}

We would like to acknowledge Martin Bland and Mike Adamson (Prozone) for their help with the match analysis data. We sincerely thank Keith Hackett and Simon Breivik (PGMOL) for their help and support in the project. The cooperation of the referees was of invaluable importance.

\section{References}

1. Bangsbo J, Norregaard L, Thorsoe F. Activity profile of competition soccer. Can J Sport Sci. 1991;16:110-116.

2. Bradley PS, Sheldon W, Wooster B, Olsen P, Boanas P, Krustrup P. High-intensity running in English FA Premier League soccer matches. J Sports Sci. 2009;27:159-168.

3. DiSalvo W, Gregson W, Atkinson G, Tordoff P, Drust B. Analysis of high-intensity activity in Premier League soccer. Int J Sports Med. 2009;30:205-212.

4. Rampinini E, Impellizzeri FM, Castagna C, Coutts AJ, Wisloff U. Technical performance during soccer matches of the Italian Serie A league: effect of fatigue and competitive level. J Sci Med Sport. 2009;12:227-233.

5. Krustrup P, Bangsbo J. Physiological demands of top-class soccer refereeing in relation to physical capacity: effect of intense intermittent exercise training. J Sports Sci. 2001;19:881-891.

6. Mallo J, Navarro E, Garcia-Aranda JM, Gilis B, Helsen W. Activity profile of top-class association football referees in relation to performance in selected physical tests. $J$ Sports Sci. 2007;25:805-813.

7. Krustrup P, Mohr M, Bangsbo J. Activity profile and physiological demands of top-class soccer assistant refereeing in relation to training status. J Sports Sci. 2002;20:861-871.

8. Mohr M, Krustrup P, Bangsbo J. Fatigue in soccer: A brief review. J Sports Sci. 2005;23:593-599.

9. Weston M, Bird S, Helsen W, Nevill A, Castagna C. The effect of match standard and referee experience on the objective and subjective match workload of English Premier League referees. J Sci Med Sport. 2006;9:256-262.

10. Reilly T. Energetics of high-intensity exercise (soccer) with particular reference to fatigue. J Sports Sci. 1997;15:257-263.

11. Lovell RJ, Barrett S, Midgley AW, Parkin G, Small K. High-Intensity activity profile in elite youth soccer match-play. Br J Sports Med. 2009;43(e2). doi:10.1136/ bjsm.2009.066894a. 
12. Edwards AM, Noakes TD. Dehydration: causes of fatigue or sign of pacing in elite soccer? Sports Med. 2009;39:1-13.

13. Weston M, Castagna C, Impellizzeri F, Rampinini E, Abt G. Analysis of physical match performance in English Premier League soccer referees with particular reference to first half and player work rates. J Sci Med Sport. 2007;10:390-397.

14. Di Salvo V, Collins A, McNeill B, Cardinale M. Validation of Prozone: A new videobased performance analysis system. Int J Perf Analysis Sport. 2006;6:108-119.

15. Cohen J. Statistical Power Analysis for the Behavioral Sciences. Hillsdale, NJ: Lawrence Erlbaum; 1988.

16. Batterham AM, Hopkins WG. Making meaningful inferences about magnitude. Int J Sports Physiol Perform. 2006;1:50-57.

17. Hopkins WG, Marshall SW, Batterham AM, Hanin J. Progressive statistics for studies in sports medicine and exercise science. Med Sci Sports Exerc. 2009;41:3-13.

18. Hopkins WG. Confidence limits and clinical chances (Excel spreadsheet). A new view of statistics. sportsci.org. Internet Society for Sports Science, sportsci.org/resource/ stats/xcl.xls. 2007.

19. Mohr M, Krustrup P, Nybo L, Nielsen JJ, Bangsbo J. Muscle temperature and sprint performance during soccer matches: beneficial effect of re-warm-up at half-time. Scand J Med Sci Sports. 2004;14:156-162.

20. Weston M, Castagna C, Impellizzeri FM, Rampinini E, Breivik S. Ageing and physical match performance in English Premier League soccer referees. J Sci Med Sport. 2010;13:96-100. 